\title{
INTEGRAL observations of the transient neutron stars SAX J1747.0-2853 and GRS 1747-312
}

\author{
Antonella Tarana ${ }^{* i}$ \\ IASF-Rome/INAF, via Fosso del cavaliere 100, 00133 Rome, Italy \\ E-mail: antonella.tarana@iasf-roma.inaf.it
}

\section{Angela Bazzano}

IASF-Rome/INAF, via Fosso del cavaliere 100, 00133 Rome, Italy

\section{Lorenzo Natalucci}

IASF-Rome/INAF, via Fosso del cavaliere 100, 00133 Rome, Italy

\section{Pietro Ubertini}

IASF-Rome/INAF, via Fosso del cavaliere 100, 00133 Rome, Italy

\begin{abstract}
We report on INTEGRAL observations of last outbursts activity of the two transients SAXJ 1747.0-2853 and GRS 1747-312. These sources show spectral state changes driven possibly by accretion instability phenomena. They are often in a quiescent state with a low luminosity of $10^{33} \mathrm{erg} \mathrm{s}^{-1}$ and abruptly show X-ray outbursts which consist of fast flux increase up to a $\mathrm{L}_{X} \sim 10^{37-38} \mathrm{erg} \mathrm{s}^{-1}$ and a slower nearly exponential decay lasting weeks or months. During the outburst the sources reveal spectral states transitions. INTEGRAL has detected two distinct outbursts from SAX J1747.0-2853 (in March 2004 and also in February 2006) and one outburst for GRS 1747-312 in February 2006. Light curves and spectral analysis have been performed with JEM-X and IBIS to show spectral state variations and to compare the sources behaviour. We report here INTEGRAL preliminary results on the outburst of March 2004 of the transient SAX J1747.0-2853 and the February 2006 outburst for GRS 1747-312. The outbursts were "soft" and the sources showed spectra extending up to $40 \mathrm{keV}$ and $20 \mathrm{keV}$ respectively, modelled by a soft blackbody emission plus a power law or Comptonization component.
\end{abstract}

7th INTEGRAL Workshop

September 8-11 2008

Copenhagen, Denmark

\footnotetext{
*Speaker.

${ }^{\dagger}$ This work has been supported by the Italian Space Agency through the grant ASI/INAF I/008/07 and I/008/06.
} 


\section{SAX J1747.0-2853}

The Neutron Star (NS) SAX J1747.0-2853, located in the Galactic Center region at about 0.5 deg from Sgr $\mathrm{A}^{\star}$, is a soft X-ray transient with an outburst recurrence time of about 1 year. It has been observed to be active in several occasions since 1998, when the source was discovered by the BeppoSAX/WFCs. This source reveals either weak or bright type of outbursts. It was detected in hard state in 1998 and in soft state during 1999 and 2000 (with BeppoSAX and XMM) and also in a low level-accretion rate state by Chandra in 2001 observation [3]. The NS nature in this LMXB system was firmly established by the detection of X-ray bursts in 2000 by BeppoSAX/WFCs [4].

\subsection{The 2004 Soft X-ray outburst}

During March 2004, RXTE and INTEGRAL detected an X-ray outburst from this source ([1], [2]). We analysed the X-ray behaviour of the source with INTEGRAL/JEM-X and IBIS starting from March 10 up to 30. Fig. 1 shows the light curve of the outburst with JEM-X in the 4-10 and $10-20 \mathrm{keV}$ and with IBIS in the $20-40 \mathrm{keV}$ band. Each point corresponds to a single pointing observation at sigma $>3$. The source flux reaches $300 \mathrm{mCrab}$ in the $4-10 \mathrm{keV}$. It was only detected few times in the IBIS $20-40 \mathrm{keV}$ and is not detected above $40 \mathrm{keV}$ band, implying that we deal with a soft X-ray outburst.

\subsection{The spectral evolution during the outburst}

We analysed the spectra from JEM-X and IBIS/ISGRI in three epochs of the outburst: at the beginning (speA, IJD=1530-1536), at the peak during the maximum in the 4-10 keV band (speB, $\mathrm{IJD}=1540-1542$ ) and soon after the peak (speC, IJD=1549-1551). Spectra have been fitted with different models. Neither of a single power law, a blackbody nor a Comptonization (compTT [5]) model alone fit the data. The best model is a soft component (diskbb or bbody) plus a hard component (represented by either compTT or power law). In all the spectral fits a high absorbtion, $\mathrm{N}_{\mathrm{H}}$ of $9 \times 10^{22} \mathrm{~cm}^{-2}$ taken from [4], is added due to the position of the source close to the Galactic Center region.

If we fit the speA with diskbb+Comptt or diskbb+po models the result is rather poor, with a $\chi_{v}^{2}$ (d.o.f) of 1.6(32) and 1.8(34) respectively. The best fit model is given by a single blackbody plus a power law and is characterised by a blackbody temperature of $1.5 \mathrm{keV}$ and a photon index of 1.9. The 4-40 keV unabsorbed flux is $8.9 \times 10^{-10} \mathrm{erg} \mathrm{cm}^{-2} \mathrm{~s}^{-1}$. The power law is needed to fit the data above $20 \mathrm{keV}$ due to hard nature of this state, but the statistics is too limited to constrain the blackbody parameters at lower energies.

For the spectra $\mathrm{B}$ and $\mathrm{C}$ the best fit is Comptt+diskbb. The spectrum speB shows an inner disk blackbody temperature of $0.6 \mathrm{keV}$ and an electron temperature of Comptonizing plasma of $3.2 \mathrm{keV}$ with an optical depth of 3 and an input seed photons temperature of $1.2 \mathrm{keV}$. The spectrum speC shows an inner disk blackbody temperature of $0.5 \mathrm{keV}$ and Comptonization with $\mathrm{kT}_{\mathrm{e}}$ of $3.6 \mathrm{keV}$, $\tau \sim 2.4$ and $\mathrm{T}_{0}=1.1 \mathrm{keV}$. The $4-40 \mathrm{keV}$ unabsorbed flux of speB and speC correspond to $5.3 \times 10^{-9}$ and $3.5 \times 10^{-10} \mathrm{erg} \mathrm{cm}^{-2} \mathrm{~s}^{-1}$ respectively. Data and model of the spectra are shown in Fig.2 and parameter model in Table 1 . 


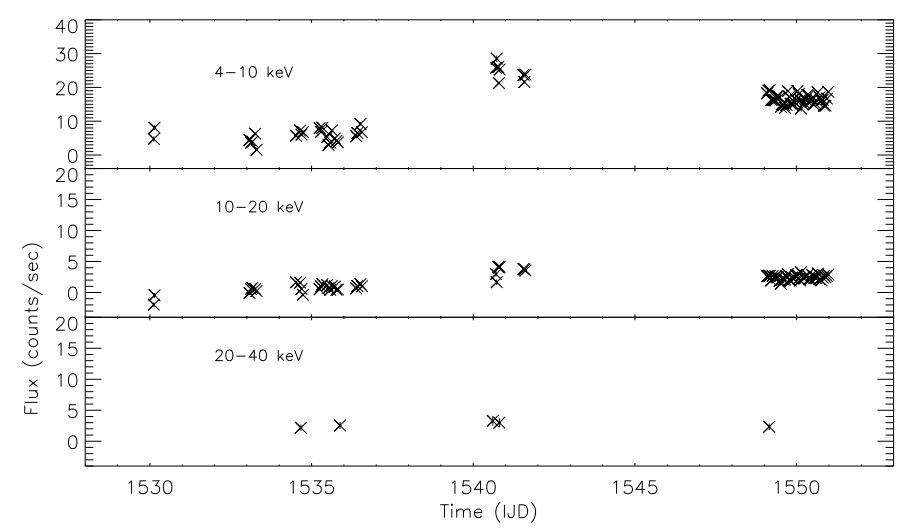

Figure 1: JEM-X and IBIS light curve of SAX J1747.0-2853 during March 2004 outburst.

Table 1: Best fit parameters of spectra of SAX J1747.0-2853.

\begin{tabular}{lccc}
\hline Parameter & speA & speB & speC \\
\hline$k T_{0}(\mathrm{keV})$ & - & 1.2 (frozen) & 1.1 (frozen) \\
$k T_{\mathrm{e}}(\mathrm{keV})$ & - & $3.2_{-0.4}^{+4.3}$ & $3.6_{-0.6}^{+1.2}$ \\
$\tau$ & - & $3.0_{-2.7}^{+0.8}$ & $2.4_{-0.9}^{+0.4}$ \\
$k T_{\text {in }}(\mathrm{keV})$ & - & $0.6_{-0.1}^{+0.1}$ & $0.5_{-0.1}^{+1.1}$ \\
$k T_{\mathrm{bb}}(\mathrm{keV})$ & $1.5_{-2.5}^{+8.1}$ & - & - \\
$\Gamma$ & $1.9_{-1.5}^{+39}$ & - & - \\
$\chi_{v}^{2}$ (d.o.f) & $1.17(31)$ & $1.17(27)$ & $1.27(30)$ \\
\hline
\end{tabular}

\section{GRS 1747-312}

GRS 1747-312 is located in a Globular Cluster (Terzan 6) at a distance of $9.5 \mathrm{kpc}$ [6]. It is a transient source and shows outburts with a recurrence time of 1-5 months and also shows total eclipses of the neutron star every $12.4 \mathrm{hrs}$ [7]. It is a type I X-ray burster and dipper and more recently superbursts events have been reported [8].

\subsection{The 2006 Soft X-ray outburst}

During February 2006, INTEGRAL detected an X-ray outburst from this source (Atel 734). We analysed the X-ray behaviour of the source with INTEGRAL/JEM-X and IBIS starting from February 9 up to March 27. Fig. 3 shows the light curve of the outburst with JEM-X in the 4-10 and $10-20 \mathrm{keV}$. The source is not detected by IBIS above $20 \mathrm{keV}$. Each point correspond to a single pointing observation. The source reaches $45 \mathrm{mCrab}$ in the $4-10 \mathrm{keV}$. 

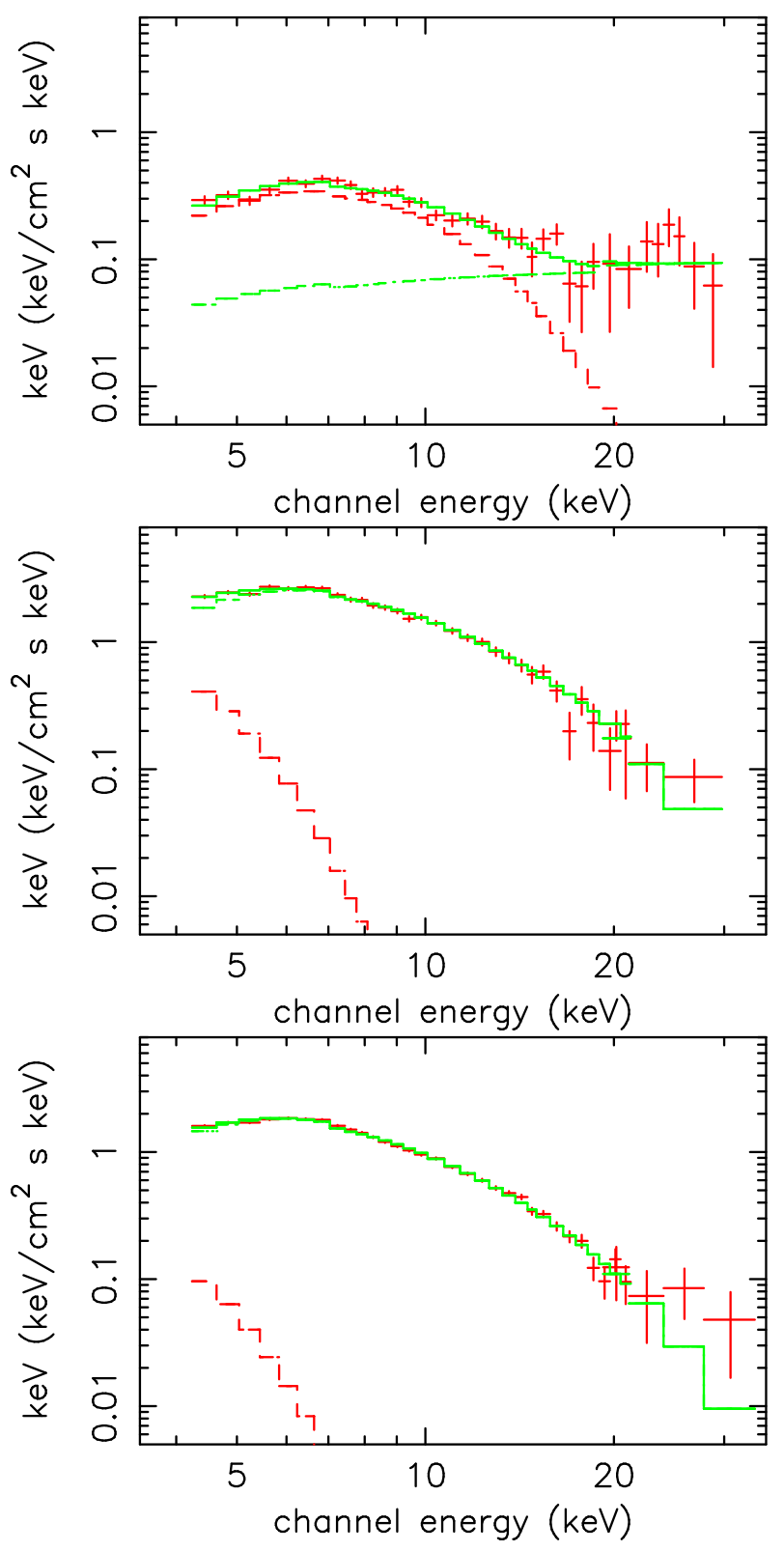

Figure 2: INTEGRAL spectra of SAX J1747.0-2853. From top to bottom: speA, speB, speC.

\subsection{The spectral evolution during the outburst}

We analysed the JEM-X spectra in two epochs of the outburst: at the peak during the maximum in the 4-10 keV band (spe1, IJD=2231.4-2242.2) and during the decay phase (spe2, IJD=2242.22253). After the spe 2 epoch, the source is detected only at an upper limit of $6 \mathrm{mCrab}$. We try to fit the spectra with different models. The single power law, blackbody and compTT alone doesn't fit the data. The best model is a soft component (diskbb) plus a hard Comptonised component (CompTT). In all the spectra fitting an absorption, $\mathrm{N}_{\mathrm{H}}$, of $1.2 \times 10^{22} \mathrm{~cm}^{-2}$ was used, as in [7]. A disk blackbody temperature of $0.5 \mathrm{keV}$ and an electron temperature of Comptonizing plasma of $2.2 \mathrm{keV}$ with an optical depth of 7.3 and an input seed photons temperature of $1.1 \mathrm{keV}$ are the parameters 


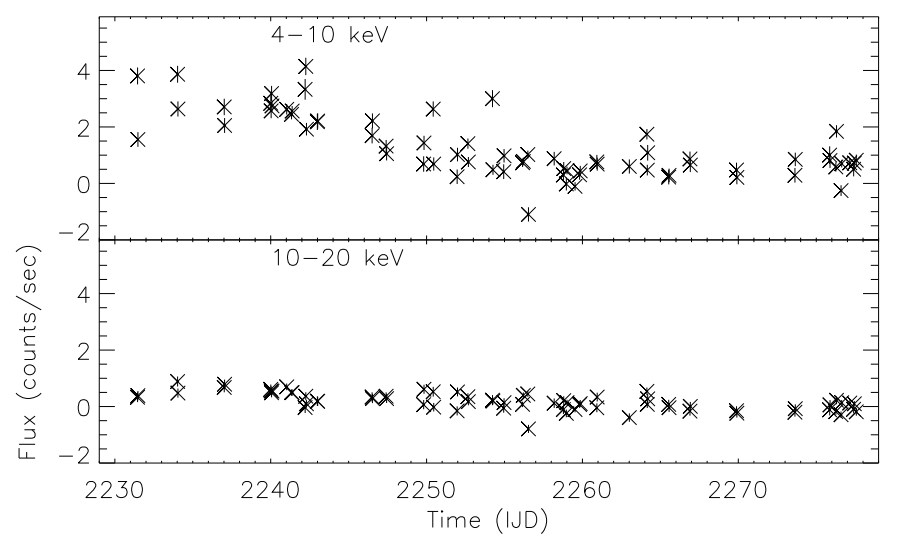

Figure 3: JEM-X light curve of GRS 1747-312 during the February-March 2006 outburst.

values for spe1. For the spectrum spe 2 a blackbody temperature of $0.3 \mathrm{keV}$ and Comptonization with $\mathrm{kT}_{\mathrm{e}}$ of $2.7 \mathrm{keV}$, a optical depth of 7.4 and a temperature of the seed photons of $0.1 \mathrm{keV}$ are obtained. The 4-20 keV unabsorbed flux of spe1 and spe2 correspond to 3.1 and $1.4 \times 10^{-10} \mathrm{erg}$ $\mathrm{cm}^{-2} \mathrm{~s}^{-1}$ respectively. Data and model of the spectra are shown in Fig. 4 and parameters model in Table 2.
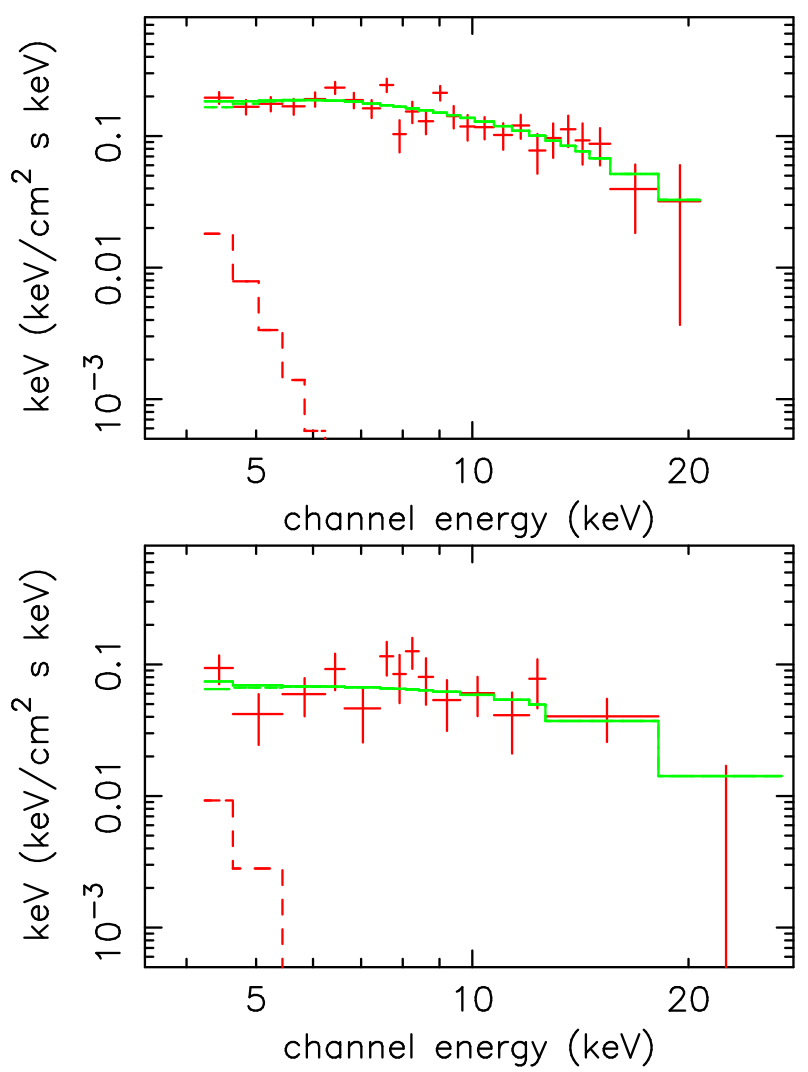

Figure 4: INTEGRAL soectra of GRS 1747-312. From top to bottom: spe1, spe2. 
Table 2: Comptt+diksbb parameters of GRS 1747-312.

\begin{tabular}{lcc}
\hline Parameter & Spe1 & Spe2 \\
\hline$k T_{0}(\mathrm{keV})$ & 1.04 (frozen) & 0.1 (frozen) \\
$k T_{\mathrm{e}}(\mathrm{keV})$ & $2.6 \pm 0.7$ & $2.7 \pm 1.2$ \\
$\tau$ & $5.6 \pm 2.5$ & $7.4 \pm 6.6$ \\
$k T_{\text {in }}(\mathrm{keV})$ & $0.5 \pm 0.8$ & $0.4 \pm 0.1$ \\
$\chi_{v}^{2}($ d.o.f) & $0.87(146)$ & $1.09(146)$ \\
\hline
\end{tabular}

\section{Discussion and conlcusions}

The INTEGRAL observations allow us to follow the behaviour of the transient SAX J1747.02853 during the 2004 outburst. It doesn't show high energy emission above $40 \mathrm{keV}$ indicating a Soft spectral state. This behaviour is similar to the one already observed in Spring 2000 by BeppoSAX [9]. In fact the three spectra analysed during the evolution of the outburst show a soft component due to a blackbody emission, coming from the accretion disk and/or the neutron star and a Comptonization (or also power law) component due to the inverse Compton scattering of the soft photons by the electron of the corona. The 4-40 keV maximum luminosity reached during the outburst correpond to $5 \times 10^{37} \mathrm{erg} \mathrm{s}^{-1}$ assuming a distance of $9 \mathrm{kpc}$ [4]. Further analysis of next outbursts detected by INTEGRAL are in progress to better characterize the source behaviour.

We also follwed GRS 1747-312 during the 2006 outburst. During the outburst it didn't show emission above $25 \mathrm{keV}$ revealing that also this source showed a "soft" outburst. Again for this source the better model is represented by a disk blackbody emisssion plus a Comptonization emission coming from an hypothetic corona surrounding the accretion disk. The 4-20 keV maximum luminosity reached during the outburst correpond to $3.37 \times 10^{36} \mathrm{erg} \mathrm{s}^{-1}$ assuming a distance of 9.5 $\mathrm{kpc}[6]$.

\section{References}

[1] Markwardt, C. B., et al. 2004, ATel 255

[2] Deluit, S. et al. 2004, ATel 256

[3] Wijnands, R., et al. 2002 ApJ 579, 422

[4] Natalucci L. et al. 2000 ApJ 543, L73

[5] Titarchuk L. ApJ 434, 570, 1994

[6] Predehl, P. et al. 1991, A\&A 246, L21

[7] In't Zand et al., 2000 A\&A 355, 145

[8] In't Zand et al., 2003 A\&A 409, 659

[9] Natalucci et al. 2004 A\&A 416, 699 